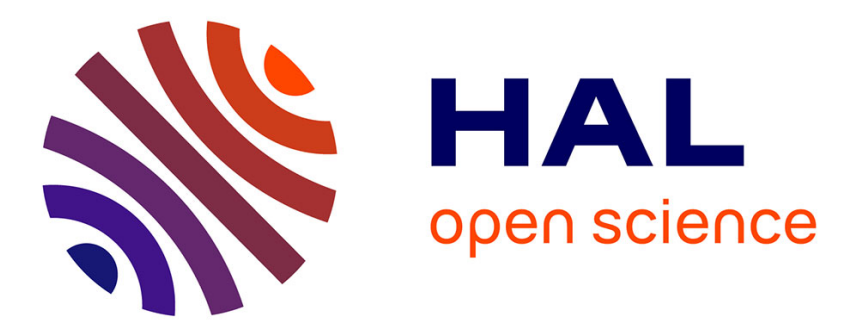

\title{
Les genres numériques de la relation
}

Christelle Combe

\section{To cite this version:}

Christelle Combe. Les genres numériques de la relation. Langage et Société, 2019, Nº167 (2), pp.51. 10.3917/ls.167.0051 . hal-02552167

\section{HAL Id: hal-02552167 \\ https://hal.science/hal-02552167}

Submitted on 23 Apr 2020

HAL is a multi-disciplinary open access archive for the deposit and dissemination of scientific research documents, whether they are published or not. The documents may come from teaching and research institutions in France or abroad, or from public or private research centers.
L'archive ouverte pluridisciplinaire HAL, est destinée au dépôt et à la diffusion de documents scientifiques de niveau recherche, publiés ou non, émanant des établissements d'enseignement et de recherche français ou étrangers, des laboratoires publics ou privés. 


\title{
Les genres numériques de la relation
}

Christelle Combe

Aix Marseille université, CNRS, LPL, Aix-en-Provence, France

christelle.combe@univ-amu.fr

\section{Introduction}

Depuis la fin du $\mathrm{XX}^{\mathrm{e}}$ siècle, les modes de communication sont profondément bouleversés par l'accès facilité à Internet et la multiplication et miniaturisation des différents terminaux nous permettant d'y accéder. De nouvelles formes de rédaction et de publication adaptées à la convergence des technologies et des pratiques sociales numériques sont ainsi en train de voir le jour et de se stabiliser. La notion de genre de discours s'avère donc toujours aussi prégnante car comme l'affirme Mikhaïl Bakhtine :

\begin{abstract}
Le vouloir-dire du locuteur se réalise avant tout dans le choix d'un genre de discours. Ce choix se détermine en fonction de la spécificité de la sphère de l'échange verbal, des besoins d'une thématique (de l'objet du sens), de l'ensemble constitué des partenaires, etc. Après quoi, le dessein discursif du locuteur, sans que celui-ci se départisse de son individualité et de sa subjectivité, s'adapte et s'ajuste au genre choisi, se compose et se développe dans la forme du genre donné. (1984 : 284)
\end{abstract}

Et, à l'heure de la numérisation généralisée de la société, après les genres écrits et les genres oraux, ce sont désormais les « genres numériques » qu'il convient de décrire, de caractériser, de typologiser. Bien que déjà documentés depuis une vingtaine d'années, les genres numériques l'ont été principalement avec une approche comparative à d'autres genres préexistant au numérique (Mourlhon-Dallies et al. 2004 ; Labbé \& Marcoccia 2005) et selon des cadres d'analyse prénumériques (Maingueneau 2013). Or, dans cet article, nous souhaitons prendre davantage en compte l'écosystème du web (Paveau 2013) car la question de la contrainte technologique dans le fonctionnement des genres en ligne est primordiale (Paveau 2017). C'est donc en envisageant plutôt la dimension socio-technolangagière que nous nous proposons de caractériser le blogue et le vlogue, deux technogenres prototypiques et emblématiques du web 2.0. Tel qu'il a été précédemment défini, le blogue est un site internet qui présente une forme éditoriale relativement stabilisée, récurrente et fortement reconnaissable: «la centralité sémiotique de l'énonciateur principal (l'auteur-éditeur du blog), la liste de liens, un calendrier permettant un accès aux archives, l'organisation verticale antichronologique des textes rédigés, l'inscription de la polyphonie dans la page par le biais des zones de commentaire » (Candel 2010 : 25). Le vlogue, quant à lui, s'est principalement développé avec la création du site d'hébergement de vidéos YouTube et se caractérise par une vidéo monologale autocentrée, augmentée de commentaires écrits (Combe Celik 2014; Combe 2017). Tous les contenus du web (un des services d'Internet) étant liés via les algorithmes qui l'organisent et la majeure partie des relations et des activités sociales passant désormais par Internet, c'est sous l'angle de la relationalité ${ }^{1}$ (Paveau 2017) et selon le récent cadre théorique de l'analyse du discours numérique que nous nous proposons de décrire, à

${ }^{1}$ Cette notion est développée en 1.3 . 
notre tour, concomitamment le blogue et le vlogue, afin de proposer en conclusion une définition du «genre numérique de la relation » et de voir les évolutions qui se dessinent. Après avoir exposé le cadre théorique et la démarche méthodologique, nous interrogerons donc la structure relationnelle de ces deux technogenres à partir de deux exemples thématiques.

\section{Notions théoriques et démarche méthodologique}

Cette étude s'ancre dans le cadre théorique postdualiste de l'Analyse du Discours Numérique qui consiste en :

la description et l'analyse du fonctionnement des productions langagières natives d'Internet, et plus particulièrement du web 2.0, dans leurs environnements de production, en mobilisant à considération égale les ressources langagières et non langagières des énoncés élaborés (Paveau 2017 : 27).

Dans cette partie, nous définirons la notion de genre numérique et plus précisément de technogenre de discours prescrits avant d'expliquer la démarche méthodologique adoptée.

\section{1. Du genre au technogenre de discours}

Comme le souligne Maingueneau (2002: 592), c'est «l'une des tâches essentielles de l'analyse du discours que de classer les discours qui sont produits dans une société » et de nombreux travaux en analyse du discours s'inscrivent dans une démarche globale de typologie des genres (Marcoccia 2016). Toutefois, le support tient un rôle fondamental dans l'émergence et la stabilisation d'un genre (Maingueneau 2016) et l'écosystème numérique s'avère un environnement technosémiodiscursif complexe qu'il convient de prendre en compte. La notion de genre de discours au sein d'un écosystème numérique devient donc pour Maingueneau (2013) dans son analyse du blogue un «hypergenre », notion reprise lors d'une précédente étude sur le vlogue (Combe Celik 2014) et pour Paveau un «technogenre de discours », c'est-à-dire :

\footnotetext{
Un genre de discours doté d'une dimension composite, issue d'une coconstitution du langagier et du technologique. Le technogenre peut relever d'un genre appartenant au répertoire prénumérique, mais que les environnements numériques natifs dotent de caractéristiques spécifiques (comme le commentaire en ligne), ou constituer un genre numérique natif et donc nouveau (comme la twittérature ou l'article de presse sous forme d'anthologie de liens ou de tweets). Le technogenre de discours est donc marqué par ou issu de la dimension technologique du discours, ce qui implique un fonctionnement et des propriétés particuliers (2017: 300).
}

Par ailleurs, selon les contraintes technologiques, les différents technogenres de discours peuvent être classés en trois catégories: le «technogenre prescrit», le «technogenre négocié »et le «technogenre produsé » que Paveau (2017) catégorise ainsi :

- le technogenre prescrit est contraint par les dispositifs technologiques, n'existe pas hors ligne, dépend entièrement des outils numériques et circule quasi exclusivement en ligne (comme par exemple le tweet) ;

- le technogenre négocié préexiste de manière stabilisée ou non dans les productions prénumériques, mais se dote en ligne de traits proprement technolangagiers et 
technodiscursifs et circule dans les univers en ligne et hors ligne (comme par exemple le e-portfolio) ;

- le technogenre produsé ${ }^{2}$ est natif d'Internet et est produit par les internautes hors des contraintes des technogenres prescrits et des routines des technogenres négociés (comme par exemple le mème).

Selon cette catégorisation, le blogue et le vlogue relèvent donc des technogenres prescrits en raison, comme nous allons le voir, du caractère contraint des dispositifs d'écriture en ligne au sein desquels ils prennent forme.

\section{2. Caractéristiques des technogenres prescrits}

En contexte numérique, les textes sont le produit d'autres textes qui les gouvernent et les mettent en forme, «architexte » pour Jeanneret et Souchier (1999), « espace d'exposition et de production discursive » pour Develotte (2006) ou «préconstruction technolangagière » pour Bibié-Emerit (2018). L'énonciation éditoriale (Jeanneret \& Souchier 2005) et l'écriture numérique (Paveau 2017) des technogenres prescrits s'effectuent donc au sein d'espaces particuliers qui les configurent. Nous décrirons ici les deux espaces dans lesquels le blogue et le vlogue généralement s'actualisent : les CMS et les plateformes interactives multimodales.

\section{2. 1. Les CMS}

Les CMS (en anglais Content Management System) sont « des systèmes de gestion de contenu des sites internet qui permettent la publication et l'organisation des contenus, ainsi que la gestion des utilisateurs et leur catégorisation en termes d'accès » (Paveau 2017 : 141). JeannePérier les définit ainsi :

Les CMS se donnent donc comme vocation de permettre à d'autres d'écrire, à partir de modèles d'écritures préétablis, façonnés par des pratiques de «maintenance » anciennes dans d'autres secteurs (presse, édition, documentation, informatique), comportant des routines, des organisations, des modes de circulations des textes déjà bien ancrés dans la société dans son ensemble ou des milieux professionnels. (2006:122)

WordPress est sans doute le CMS le plus connu et le plus utilisé aujourd'hui. Créé en 2003, il est gratuit et Open Source. Il revendique $31 \%$ du web, des blogues personnels aux plus gros sites d'actualités en ligne. Élu meilleur CMS en 2007, ses développeurs et utilisateurs se réunissent lors de rencontres mensuelles dans le monde entier et depuis 2008, il existe une communauté Wordpress francophone ${ }^{3}$ qui organise un Wordcamp Paris annuel depuis 2011. C'est sur ce CMS que nous nous appuierons pour illustrer notre propos.

\section{2. 2. Les plateformes interactives multimodales}

Les plateformes interactives multimodales sont définies par Herring ainsi :

Les plateformes interactives multimodales (désormais PIM) permettent aux utilisateurs de médias sociaux de commenter un contenu multimodal par des canaux multiples sur un seul site - et même au sein d'un même fil ou d'une même conversation. Une PIM comprend au

\footnotetext{
${ }^{2}$ De «produsage », néologisme qui désigne le fait de produire de nouveaux contenus, d'inventer de nouveaux usages à partir des contenus déjà fournis par les espaces d'écriture en ligne (Paveau 2017).

${ }^{3}$ Voir en ligne : <https://wpfr.net/>.
} 
minimum du texte plus un autre mode (audio, vidéo et/ou graphique) ; les modes peuvent être synchrones ou asynchrones. (notre traduction)

Interactive multimodal platforms (henceforth, IMPs) allow social media users to comment on multimodal content via multiple channels on a single website-and even within a single thread or conversation. An IMP minimally involves text plus one other mode (audio, video, and/or graphics); the modes may be synchronous or asynchronous. (2015:2)

YouTube, créée en 2005, est l'une des premières plateformes interactives multimodales. Rachetée en octobre 2006 par Google, elle compte en 2018 plus d'un milliard d'utilisateurs, ce qui représente un tiers d'Internet. Dans un ouvrage consacré à cette plateforme, Burgess et Green précisent :

L'innovation initiale était une innovation technologique (mais pas que) : YouTube faisait partie des nombreux services concurrents visant à supprimer les obstacles techniques au partage généralisé de vidéos en ligne. Le site web fournissait une interface très simple et intégrée dans laquelle les utilisateurs pouvaient télécharger, publier et visionner des vidéos en streaming sans connaissances techniques poussées, et dans le respect des contraintes technologiques des logiciels de navigateur standard et d'une bande passante relativement modeste. (notre traduction)

The original innovation was a technological (but non-unique) one: YouTube was one of a number of competing services aiming to remove the technical barriers to the widespread sharing of video online. The website provided a very simple, integrated interface within which users could upload, publish, and view streaming videos without high levels of technical knowledge, and within the technological constraints of standard browser software and relatively modest bandwidth. (2009: 1)

C'est sur cette plateforme, désormais incontournable, que nous nous appuierons pour illustrer notre propos.

\section{3. Quelle démarche pour étudier les technogenres?}

La relationalité, telle que définie par Paveau, est une des caractéristiques des discours produits dans un environnement numérique connecté. Elle se manifeste à trois niveaux :

relation avec les autres technodiscours du fait de la structure hypertextuelle du web; relation avec les appareils du fait de la nature composite des technodiscours, littéralement coproduits dans la machine ; relation avec les scripteurs et les (écri)lecteurs qui passe par la subjectivité de la configuration des interfaces d'écriture et de lecture. (Paveau 2017 : 285)

Après avoir envisagé l'énonciation numérique de ces deux protogenres que sont le blogue et le vlogue, c'est à partir de cette définition (et principalement du premier et dernier niveau) que nous interrogerons la structure relationnelle du blogue et du vlogue.

En outre, étudier les discours natifs en ligne «c'est-à-dire dans un écosystème numérique connecté » (Paveau 2015 : 3) nécessite d'adopter une posture de recherche particulière et de repenser les dispositifs méthodologiques. Les données que nous avons étudiées n'ont pas été à proprement parler «récoltées », elles ont au contraire été observées et explorées in situ (Combe 2016), elles correspondent aux productions technolangagières en ligne, dans toute leur variété (discours, technographismes, productions multimédiatiques, etc.). En outre, ces observables ayant pour caractéristique d'être tout à la fois massives et labiles 
(Develotte 2012), dans une démarche qualitative et descriptive, nous n'avons pas cherché à être exhaustive mais plutôt à étudier deux corpus échantillons. Le formatage technologique supprimant les différences formelles, un exemple peut suffire à une étude du discours numérique natif. Parmi la multitude de blogues et de vlogues, nous avons opéré un choix et avons retenu un blogue de voyage «Votre tour du monde ${ }^{4}$ » et un vlogue d'éducation politique et citoyenne "Osons causer ${ }^{5}$ », deux thématiques qui nous semblent caractéristiques de la nouvelle civilisation numérique (Doueihi 2011) et des concepts de nomadisme et de liberté d'expression qu'elle véhicule dans l'imaginaire collectif.

Enfin, étudier ces technodiscours n'est pas sans soulever des questions éthiques et juridiques (Develotte 2012). Nous nous référons pour cela aux prescriptions de Côté (2012) et Combe (2016). Ainsi, compte tenu du fait que les échantillons étudiés sont accessibles publiquement à tout internaute et considérant que la recherche ne relève pas d'une situation délicate mais en l'occurrence d'une analyse technosémiodiscursive à visée typologique, nous faisons le choix de ne rien anonymiser (toutes les captures d'écran ayant été prises à partir de notre propre compte) et de renvoyer le lecteur vers les observables si possible par des liens intégrés dans une revue en ligne et d'illustrer notre propos par des captures d'écran. Les figures 6 et 7 sont des montages récapitulatifs réalisés à partir de captures d'écran qui devraient permettre au lecteur de suivre aussi plus aisément notre propos.

\section{L'énonciation numérique de deux technogenres prescrits}

Dans cette partie, nous présenterons les caractéristiques technosémiodiscursives de l'énonciation numérique à partir des notions d'espaces d'exposition et de production discursive, de représentation de soi, d'extimité et de web-intimité.

\section{1. Espace de production discursive et scène générique contrainte}

L'espace d'exposition discursive est l'environnement composé des discours auquel est exposé un acteur. L'exposition discursive conditionne la production discursive : c'est en effet en fonction d'un espace d'exposition discursive spécifique que s'effectue la production d'un nouveau discours de la part d'un locuteur, sujet psychologique et acteur socialement situé (Develotte 2006). Le CMS et la plateforme YouTube sont respectivement les espaces d'exposition et de production discursive d'un blogue et d'un vlogue et nous allons voir comment ils contraignent et inspirent l'écriture numérique.

Comme nous pouvons l'observer dans la figure 1 ci-dessous, l'internaute qui souhaite créer un blogue sur Wordpress se trouve immédiatement confronté à un discours l'encourageant à créer son site sur cette plateforme en particulier. Ce discours est d'ordre incitatif ainsi que le suggère la forme impérative puis infinitive du verbe «créer» répété par trois fois en trois formats différents dès l'espace initial de production discursive du CMS WordPress. Il est aussi d'ordre publicitaire: la plateforme est largement promue au travers d'expressions hyperboliques comme «la vitrine de millions d'entreprises et de boutiques en ligne » ou encore «la plate-forme la plus populaire au monde». L'internaute est également interpellé

\footnotetext{
${ }^{4}$ Voir en ligne : <www.votretourdumonde.com>.

${ }^{5}$ Voir en ligne : <https://www.youtube.com/channel/UCVeMw72tepFl1Zt5fvf9QKQ>.
} 
non seulement par le pronom d'adresse dans l'expression « un site web qui vous ressemble », mais également par le regard et le sourire complices que lui adresse le personnage «monsieur Tout-le-monde » tenant le téléphone portable.

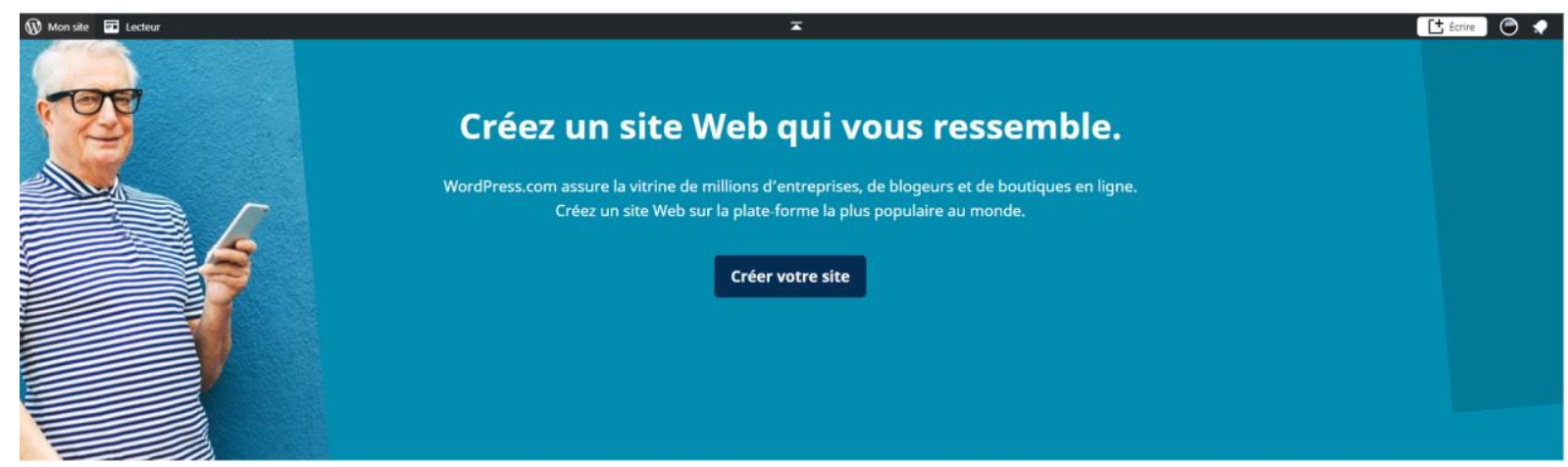

Figure 1 : Espace initial de production discursive du CMS Wordpress

Engagé dans une action conjointe d'écrilecture avec la plateforme - de «Créez », nous passons à «Créons »- l'internaute doit répondre à un questionnaire proposé par l'interface qui lui suggère des idées s'il venait à en manquer : "Comment aimeriez-vous appeler votre site ? Par exemple Dans la cuisine de Mélanie »; "Quel sera le thème de votre site ? Par exemple Mode, voyage, etc. », avant de suivre les deux étapes initiales supplémentaires : l'attribution d'un nom et d'une adresse, puis le choix d'un plan d'abonnement.

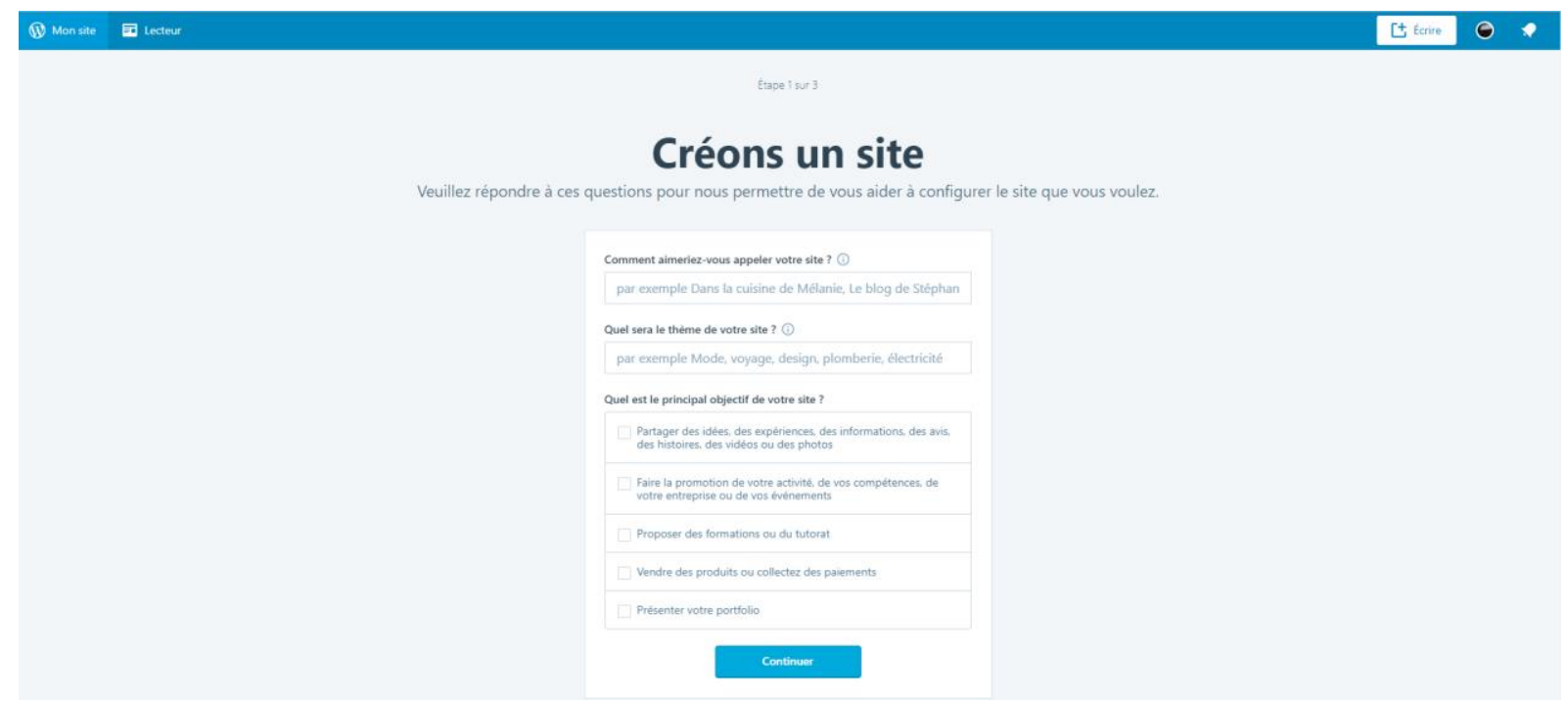

Figure 2 : Espace initial (2) de production discursive du CMS Wordpress

L'internaute se retrouve ensuite face à une interface qui «fête » littéralement la création du blogue ou site ${ }^{6}$, comme le suggère le graphisme composé d'étoiles, de ronds et traits colorés au-dessus de l'affirmation «Votre site a été créé », puis liste les actions supplémentaires à exécuter. La liste de configuration montre, qu'à cette étape, 5 étapes sur 11 ont été réalisées. Des boutons/actions «Allez-y ! » à côté de chacune des consignes non encore effectuées de la

${ }^{6}$ La distinction entre l'un et l'autre n'existe plus vraiment. 
liste de configuration comme "Charger une icône du site », "Créer une description » ou encore «Personnaliser votre page de contact» incitent fortement l'écrilecteur à poursuivre la configuration.

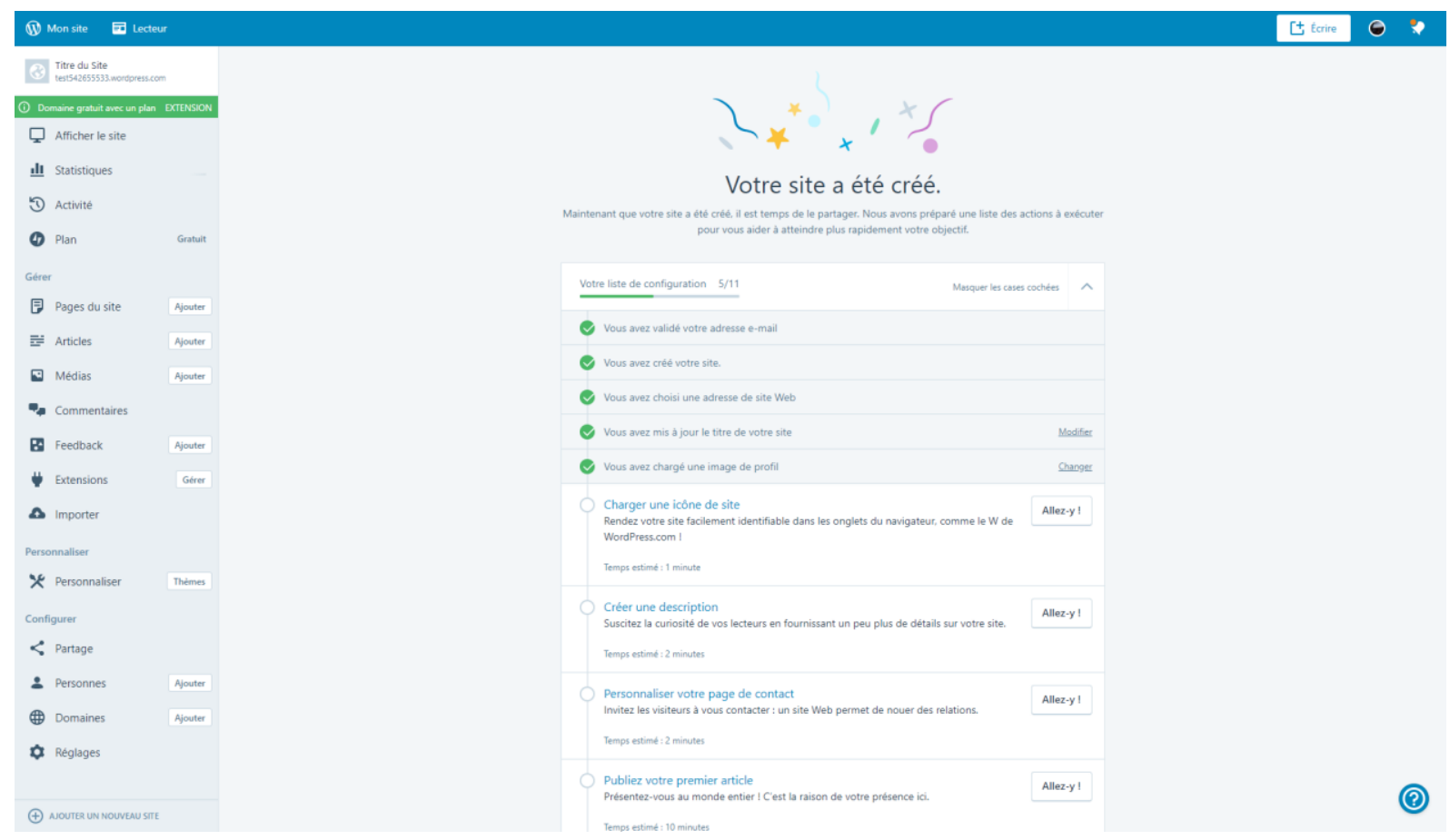

Figure 3 : Espace quasi final de production et de gestion discursive du CMS Wordpress

Sur la gauche, un menu aux nombreux items iconotextuels (l'icône d'une petite page est doublée du texte «Pages du site» et suivi d'un bouton incitatif «Ajouter») invite l'écrilecteur à la création, puis l'édition d'une page par exemple. S'ouvre alors un éditeur de textes de type similaire à celui que proposent les logiciels courants de messagerie ou de traitement de texte. Nous observons donc que c'est dans cette interlocution avec la machine qui suggère des actions discursives au blogueur en devenir que la genèse discursive du blogue se réalise.

La vidéo est au cœur du technogenre du vlogue, l'action d'importer une vidéo ou d'effectuer une diffusion en direct est donc une action centrale et lorsque l'internaute souhaite importer une vidéo, c'est également tout un dispositif iconotextuel qui oriente son action. 


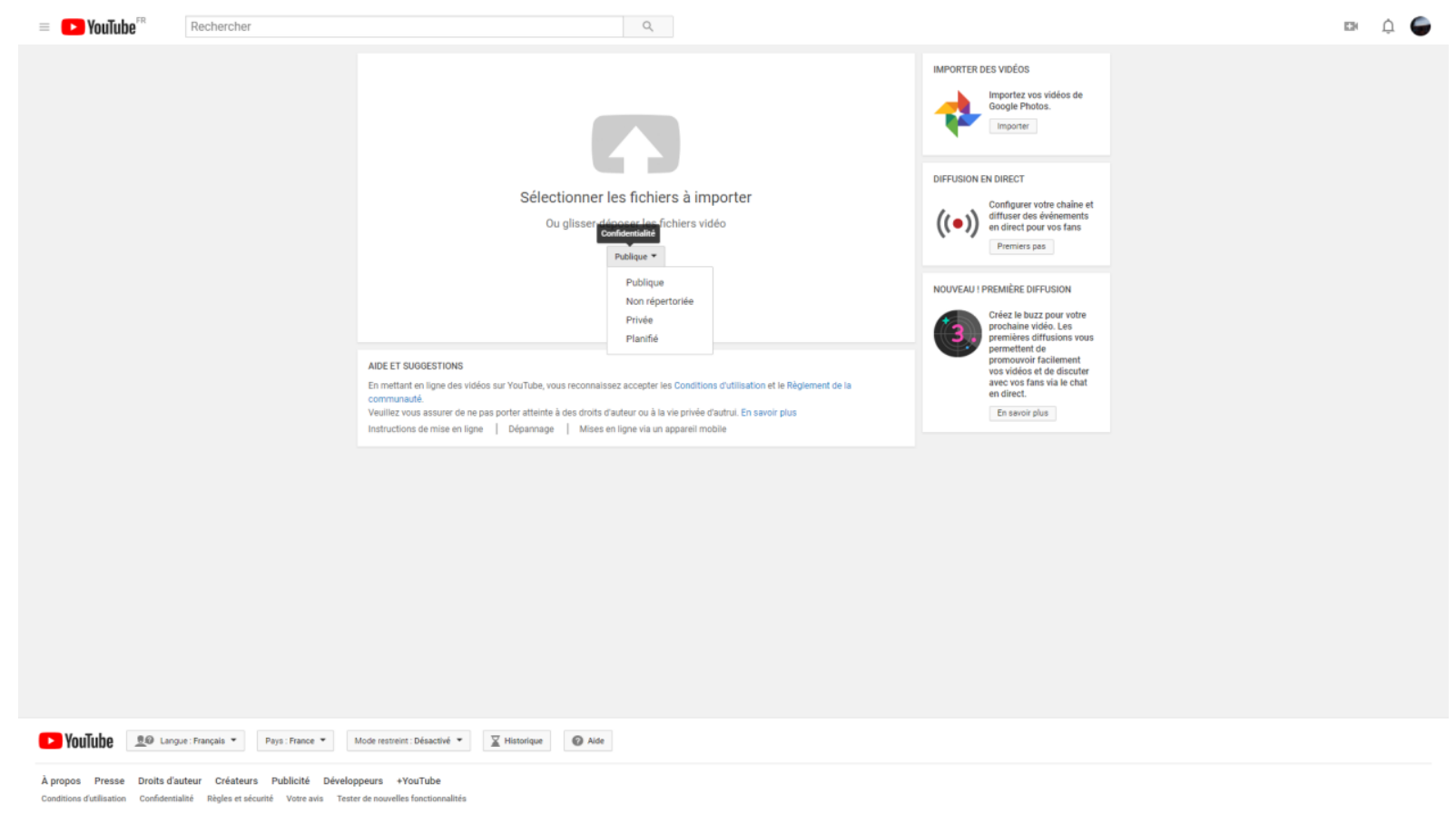

Figure 4 : Espace de production discursive de la plateforme YouTube

Une fois sa chaîne créée et ses premières vidéos importées, c'est un espace d'exposition discursive similaire qui est proposé sur la plateforme YouTube. Un menu à gauche permet d'effectuer un certain nombre d'opérations de création, mais aussi de gestion, interface qui s'enrichit et se complexifie régulièrement avec le temps.

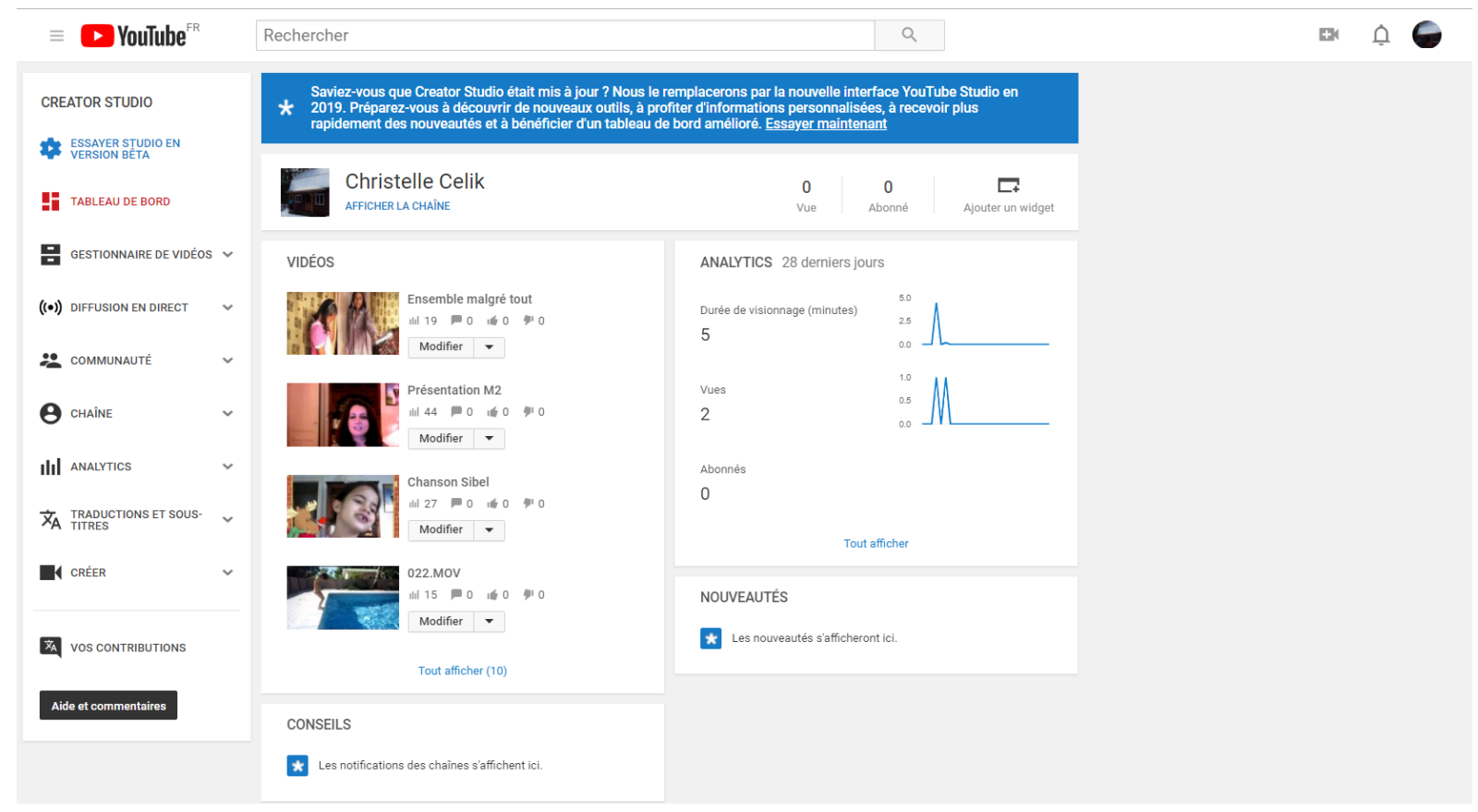

Figure 5 : Espace de production et gestion discursive de la plateforme YouTube

Le blogueur ou le vlogueur se trouvent ainsi exposés à des discours qui vont les aider à produire d'autres discours qu'ils pourront ensuite gérer au sein du CMS ou de la plateforme. 
Aux espaces d'exposition et de production discursives, nous proposons donc d'ajouter la notion d' « espaces de gestion discursive » propres à ces plateformes.

\section{2. La représentation de soi}

La Représentation de soi étant définie comme composée des signes observables à l'écran qui manifestent l'utilisateur, son agencement sémiotique est présenté à la suite : il est composé d'un embrayeur qui désigne l'utilisateur associé à des éléments qui connotent sa personnalité. (Georges 2009 : 3)

Dans un blogue ou dans un vlogue, la représentation de la figure de l'énonciateur apparaît au travers de plusieurs éléments dont principalement l'autonomination et l'avatar associé. Le pseudonyme relève de l'identité numérique et assure deux fonctions importantes de la sociabilité en contexte numérique : l'identité et l'identification. Le pseudonyme que le blogueur ou vlogueur s'attribuent à l'ouverture de leur compte relève de l'identité déclarative, c'est-à-dire un ensemble d'informations saisi dans le cadre proposé par le Système (Georges 2009). Il est important de noter que YouTube ou Wordpress peuvent être associés à un compte Google, l'autonomination peut donc se faire automatiquement à partir de celle donnée pour le compte Google. À ce nom choisi vient généralement s'ajouter une représentation visuelle, un avatar. La combinaison pseudonyme et avatar sont donc les éléments de la représentation de soi que le blogueur ou le vlogueur donnent à montrer à ses interlocuteurs.

Dans le cas du blogue exemple, l'énonciateur numérique est «Bruno », fondateur du blogue, il est présenté comme « un passionné de voyage depuis son plus jeune âge, qui a habité dans de nombreuses villes partout dans le monde et qui ne cesse de bouger ». La page « À propos » lui est consacrée. On l'y découvre au travers de texte, de photos et de vidéos. Afin de se présenter, Bruno a choisi comme pseudonyme son prénom et comme avatar une photographie de lui avec son reflet dans un train (voir figure 6). Le titre du blogue et le logo associé (voir figure 6) «Votre Tour du Monde » met cependant davantage l'accent sur l'interlocuteur qui se trouve embarqué « dans » et incité «à » une aventure qui au départ est celle de Bruno. 


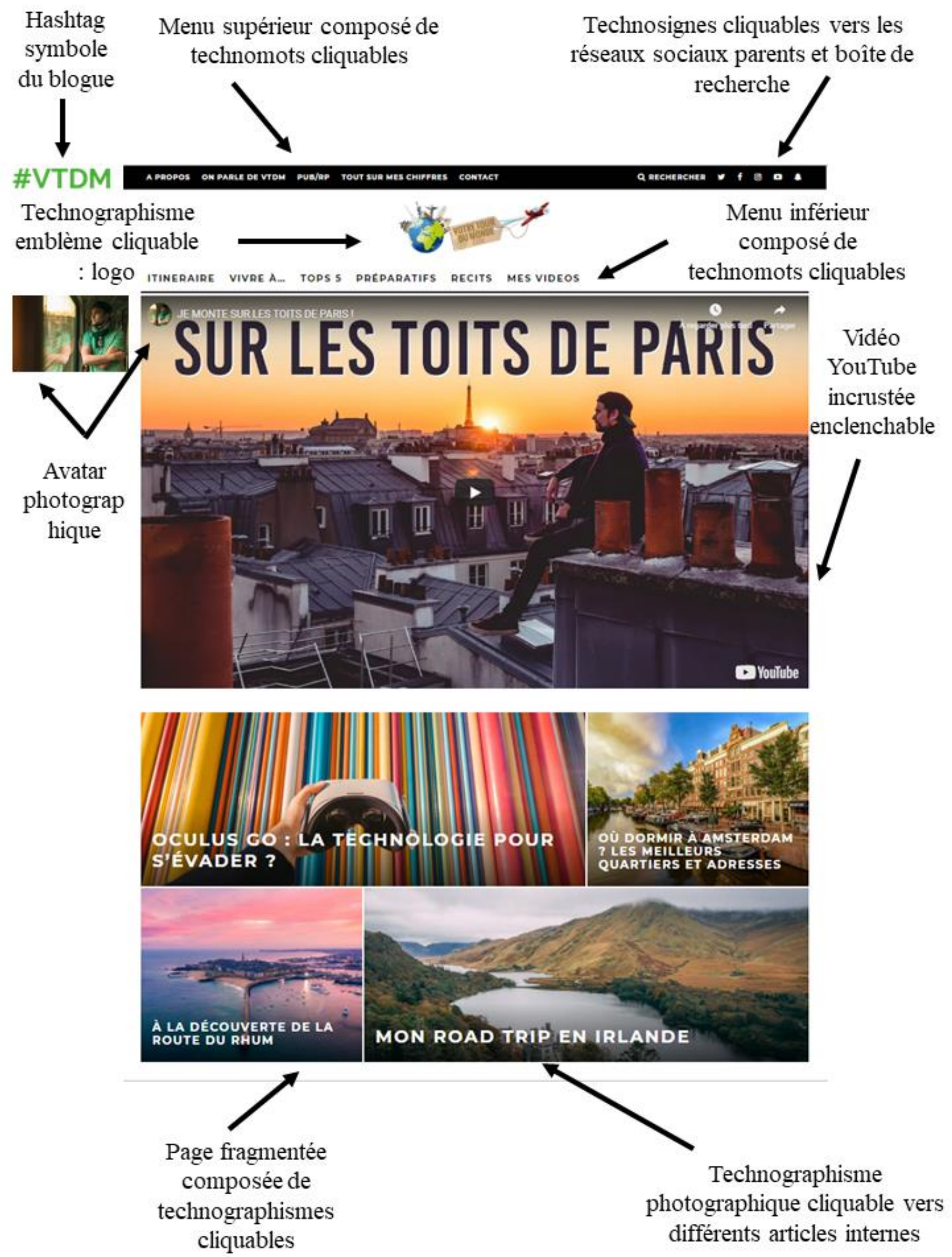

Figure $6:$ Structure relationnelle du blogue (montage sur capture d'écran)

Dans le cas du vlogue, «Osons causer» est le pseudonyme d'une équipe de trois jeunes hommes Ludo, Xavier et Stéphane, étudiants en sciences humaines et sociales. Les traits graphiques et morphologiques, comme la forme impérative à la première personne du pluriel, 
d' «Osons Causer», marquent une auctorialité groupale et engageante pour l'auditoire, associée à une injonction à prendre la parole. On se retrouve ici face à un ethos collectif qui veut se constituer en entité unifiée et agissante. Le pseudonyme s'apparente à un slogan, voire un logo, et relève finalement d'une forme de marque autodéposée participant d'une autopromotion revendicatrice (voir figure 7).

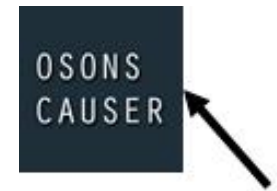

Technographisme

emblème : avatar

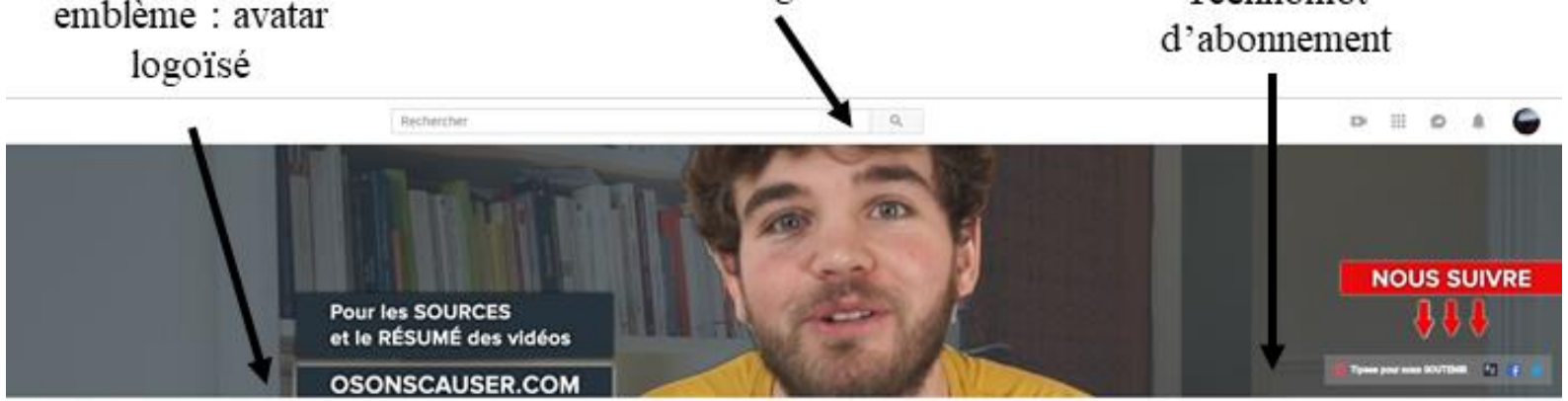

Bandeau de la chaîne : le vlogueur Ludo avec en fond la bibliothèque emblème de la scénographie du

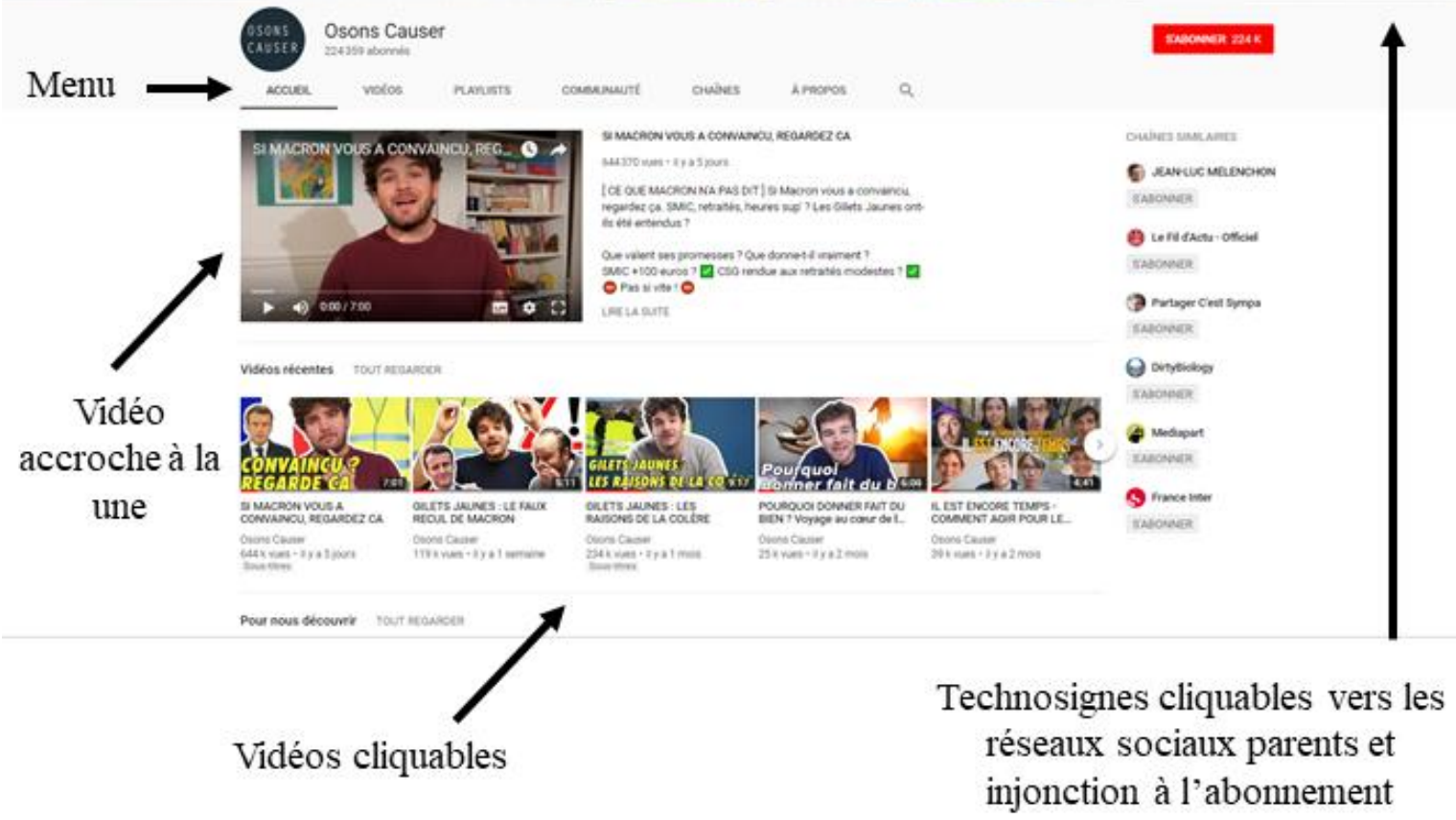

Figure 7 : Structure relationnelle du vlogue (montage sur capture d'écran)

Dans ces deux exemples, cette représentation de soi participe de la construction de l'ethos de pro-am (professionnel-amateur) (Leadbeater \& Miller 2004), c'est-à-dire un expert autodidacte, citoyen-acteur, créateur à part entière (Flichy 2014), qu'il soit individuel ou représente un collectif.

\section{3. Extimité du blogue et web-intimité du vlogue}


Le blogue et le vlogue se caractérisent comme des genres natifs de l'extériorisation de soi. Ils sont en quelque sorte dédiés aux pratiques de l'extime.

\begin{abstract}
L'extimité consiste sur internet, et tout particulièrement sur les réseaux sociaux numériques du web participatif, en l'extériorisation de l'intimité des internautes à des fins de validation de l'image de soi [...]. Le processus d'extimité vise à obtenir une validation de la part d'autrui en sollicitant sa reconnaissance (Granjon 2012). Ainsi l'extimité apparaît comme une pratique d'exposition de soi et de dévoilement de soi destinée à consolider et à s'approprier son image, ainsi qu'à augmenter son capital social (Casilli 2013) (Paveau 2017 : 185).
\end{abstract}

Souvent le blogue a été associé au journal intime, car défini comme une page web fréquemment modifiée dans laquelle les entrées datées sont répertoriées dans l'ordre chronologique inverse (Herring et al. 2005) et présentant en outre une rhétorique épistolaire (Deseilligny 2008). Quant aux vlogues, à propos des pratiques autoscopiques, Thély (2012) parle de web-intimité.

Dans le blogue, les pratiques de l'extimité se font au travers de récits intimistes, de conseils et de retour d'expérience à la première personne. L'auteur se confond avec le narrateur et le héros de ces aventures. Les pratiques extimes sont aussi fondamentalement multimédiatiques, l'avatar est le premier marqueur d'extimité : il joue un rôle dans la mise en scène du blogueur en tant que voyageur «tourdumondiste » et en mouvement (voir figure 6). La plupart des textes du blogueur sont par ailleurs enrichis d'autoportraits dans des contextes de voyages divers et de vidéos dans lesquelles il se met en scène. Dans le détail de ses différents voyages, lorsqu'il donne des conseils pratiques complets, par exemple sur les préparatifs d'un tour du monde (comme dans les onglets «Budget tour du monde», «Quelle banque choisir en voyage », «Assurance tour du monde », « Matériel photo et vidéo en voyage »), au sein d'un ensemble multimédiatique parfaitement maitrisé et de grande qualité, Bruno fait preuve d'un professionnalisme certain. La tonalité du texte reste cependant modeste et amicale, comme en témoignent les nombreuses adresses aux lecteurs, les émoticônes ou comme en atteste, par exemple, dans le billet « 10 conseils pour réussir ses photos de voyage », l'extrait suivant : «Avant de commencer, je préfère le dire tout de suite: je ne suis pas photographe professionnel! Je n'ai pas cette ambition et je n'estime pas du tout en avoir le niveau. Mais j'ai appris la photo sur le tas et je pense que mon langage simple pourra être utile pour tous les initiés qui souhaitent progresser. »

En ce qui concerne le vlogue, la web-intimité n'apparaît pas nécessairement dans l'avatarlogo qui renvoie davantage au collectif engageant, comme nous l'avons souligné précédemment, mais plutôt dans la mise en scène du locuteur principal, à savoir Ludo, qui apparaît dans le bandeau de la chaîne (voir figure 6) et surtout la scénographie de chacune des vidéos lorsqu'il s'adresse à son auditoire depuis la même pièce de leur colocation étudiante. Ludo, en t-shirt ou pull, est généralement assis sur un canapé de type clic-clac, une bibliothèque tapissée de livres peu ordonnés constitue le fond du décor, un détail vient parfois rappeler la thématique de la vidéo. L'ouverture et la clôture sont ritualisées sur un ton familier, visant à établir un lien de connivence avec l'auditoire (Combe à paraître en 2019). La vulgarisation, à partir de sources diverses, de thématiques politiques, économiques, 
sociales du moment qu'il effectue est pourtant empreinte d'éléments de didacticité et relève d'un discours pédagogique élaboré (Combe à paraître en 2019).

L'ethos qui se dégage dans nos deux exemples est un ethos de simplicité mêlé à de la compétence, l'ethos du pro-am se réapproprie des sphères de l'activité sociale traditionnellement dévolues aux professionnels, ici le voyage ou la politique. Les pratiques extimes de narration de soi dans le blogue et de web-intimité dans le vlogue se transforment en sources d'inspiration (marchande ou engagée) : une forme d'autopromotion du pro-am et de ses valeurs destinée à capter l'attention d'un auditoire encore non défini.

\section{La structure relationnelle entre technodiscours : navigation, circulation et affiliation}

Comme Crystal (2006) le soulignait déjà, le blogue a introduit une nouvelle ère d'interactivité. Et c'est premièrement dans la mise relation avec les autres technodiscours au travers des hyperliens, des technomots, technosignes et technographismes mais également des tags et hashtags, que s'observe cette interactivité.

\section{1. Hyperliens et technomots}

La mise en relation avec les autres discours du web est un des traits structuraux des discours numériques natifs, notamment du fait de la structure hypertextuelle du web. Dans le blogue «Votre Tour du Monde», nous observons un dispositif hypertextuel particulièrement complexe dès la page d'accueil.

En effet, c'est tout d'abord à partir de deux menus dans la partie supérieure du blogue et toujours visibles sur l'ensemble des pages que l'écrilecteur pourra naviguer au sein du blogue. Chaque mot du menu est donc un « technomot», soit « un élément lexical simple ou composé cliquable, c'est-à-dire dirigeant l'écrilecteur d'un texte-source vers un texte-cible, relevant d'une autre situation énonciative » (Paveau 2017 : 337). Compte tenu de la qualité esthétique générale du blogue et donc potentiellement des compétences du blogueur en termes d'édition numérique, l'URL (l'adresse internet de la page cible) est masquée et directement intégrée à chaque élément lexical du menu. Au sein des pages (ou articles ou billets) du blogue, davantage composées de parties textuelles, certains éléments syntagmatiques sont de couleur verte et au passage de la souris s'autosignifient comme unité cliquable par un trait de soulignement (voir figure 6).

Dans le vlogue, les hyperliens sont moins présents, on retrouve un menu supérieur avec des éléments lexicaux cliquables permettant de naviguer au sein des différentes pages de la chaîne YouTube «Osons causer ». Des URL non intégrées et non cliquables sous sa forme codées informatiquement vers les sites extérieurs d' «Osons causer » apparaissent dans la section « À propos » comme par exemple « Notre LIVRE : http://www.onvautmieuxqueca.com/» ainsi que des URL insérées dans un mot et colorées en bleu pour signifier leur cliquabilité.

\section{2. Technosignes et technographismes}

De technosigne, Paveau donne la définition suivante :

Le technosigne relève de l'icône, mais comme le technomot, il est cliquable et peut réaliser certains actions discursives : il s'agit par exemple des boutons sociaux (boutons de partage ou d'accès aux réseaux sociaux). (2017: 337) 
Au sein du blogue, nous observons cinq technosignes dans le menu en haut à droite renvoyant aux autres réseaux sociaux du blogueur, un compte Twitter, Facebook, Instagram, YouTube et Snapchat, répétés pour les quatre premiers à droite dans un bandeau vertical sur les pages autre que la première et dans le pied de page du blogue avec l'injonction «Suivez moi ! (sic) ». Par ailleurs, sur la première page notamment mais aussi sur d'autres articles, un ensemble de visuels cliquables, des technographismes, sont observables : des vidéos et des photos (associées parfois à du texte) qui renvoient elles-mêmes à une page au sein même du blogue ou vers une vidéo du blogueur déposée sur sa chaîne YouTube mais intégrée au blogue et qui s'enclenche au clic.

Dans le vlogue, nous observons également une mise en réseau du discours à travers les différents sites et réseaux parents par des boutons cliquables. À la chaine initiale, sont principalement associés quatre autres sites ${ }^{7}$ que l'auditoire est d'ailleurs vivement enjoint à suivre : une page Tipeee pour le financement participatif, un site explicatif du projet, une page Facebook et un compte Twitter (voir figure 7). Dans le vlogue, c'est également souvent en fin de vidéo que l'on retrouve des technosignes afin d'inciter l'écrilecteur soit à aller sur les sites parents extérieurs comme la page Facebook ou la page Tipeee soit à s'abonner par une gestuelle du vlogueur au sein de la vidéo renvoyant au bas de l'écran pour inciter l'interlocuteur à cliquer sur le bouton rouge d'abonnement.

Dans le blogue, d'autres technosignes apparaissent distincts des autres à la fin de chaque article, afin de partager le contenu du blogue sur le réseau personnel de l'écrilecteur. Nous retrouvons les principaux réseaux sociaux comme Facebook et Twitter mais aussi LinkedIn, le réseau social professionnel, et Pinterest, le site de réseautage social et partage d'intérêt.

Dans le vlogue, cette fonction apparaît à travers le technosigne d'une petite flèche associée au mot « partager » qui ouvre une boîte proposant soit d'intégrer le lien, soit de le copier, soit de l'envoyer par mail, soit de le partager directement au sein de réseaux sociaux variés. L'écrilecteur peut également interagir avec le contenu discursif en ajoutant des traductions aux vidéos par exemple.

Les technosignes ont donc deux vocations principales, à savoir permettre à l'écrilecteur de circuler au sein des discours du blogueur ou vlogueur sur les sites parents, chaque microdiscours du même énonciateur trouvant écho et place au sein d'un macro-univers discursif, et également de diffuser à l'extérieur le contenu du blogue ou du vlogue sur son propre réseau sous la forme d'un technodiscours rapporté. Discours et énonciateurs sont ainsi liés dans un même mouvement convergent.

\section{3. Tags et hashtags}

Paveau définit le hashtag comme :

${ }^{7}$ Voir en ligne : <https://www.youtube.com/channel/UCVeMw72tepFl1Zt5fvf9QKQ> ;

$<$ http://bit.ly/tipeeeosonscauser > ;

$<$ http://www.osonscauser.com> ;

$<$ http://www.facebook.com/osonscauser > ;

$<$ https://twitter.com/osonscauser $>$. 
un segment langagier précédé du signe \#, utilisé originalement sur le réseau de microblogging Twitter, mais adapté sur d'autres plateformes comme Facebook notamment. Cette association en fait un tag cliquable, inséré manuellement dans un tweet et permettant d'accéder à un fil qui rassemble l'ensemble des énoncés contenant le hashtag. (2017 : 197)

Cette forme technolangagière a une fonction essentiellement sociale. Nous observons dans le menu en haut à gauche sur le blogue « Votre Tour du Monde » le hashtag \#VTDM qui ressort en vert, signe de sa cliquabilité. Les usagers peuvent ainsi en ajoutant ce hashtag à leur post sur les réseaux sociaux, citer leur source, s'affilier à ce blogue. Ils peuvent également, en rentrant ce même hashtag dans un moteur de recherche, retrouver l'ensemble des posts sur Internet qui l'auront cité. Le technodiscours est aussi investigable plus facilement.

Nous avons donc pu observer que la relation aux autres technodiscours se faisait principalement à partir des hyperliens et technomots, des technosignes et technographismes et des hashtags.

La structure relationnelle du blogue et du vlogue apparaît comme une structure rhizomique tournée vers une discursivité interne, au sein même du blogue et du vlogue, à partir des technomots des menus, des technographismes ou des hyperliens. Puis, cette structure s'étend d'une part vers une discursivité externe entre les principaux réseaux sociaux parents du blogue ou du vlogue par des technosignes (comme Facebook, Twitter, Instagram, Snapchat, YouTube, Tipeee ou un site) et enfin d'autre part vers des sites externes via les réseaux sociaux des écrilecteurs par technodiscours rapporté.

\section{La structure relationnelle entre écrilecteurs : appréciation, échanges et communauté}

C'est deuxièmement dans la relation entretenue avec les écrilecteurs au travers des boutons d'appréciation, au sein des commentaires ouverts aux internautes qui les suivent et des échanges plus ou moins nombreux qu'ils ont avec eux (Barton \& Lee 2013) et dans la formation d'une communauté sociotechnodiscursive que s'exprime aussi la relationalité du blogue et du vlogue.

\section{1. Les boutons d'appréciation}

La relation entre blogueur, vlogueur et écrilecteur passe tout d'abord par les boutons d'appréciation, à savoir les différents technosignes qui permettent notamment une opération discursive explicite comme celle d' «aimer» ou pas un contenu. Si le geste d' «aimer» un contenu sur les réseaux sociaux est polysémique et peut recouvrir plusieurs significations comme «j'aime bien ce que tu publies, je suis là, j'ai lu, je t'envoie un petit salut, je te dis bonjour, je soutiens l'ouverture de ton compte, etc. » (Paveau 2017 : 320), dans le cas du blogue et du vlogue, ces énoncés de geste manifestent la plupart du temps une appréciation et évaluent un contenu.

Ainsi, sur la plateforme YouTube, pour chaque vidéo à côté du nombre de vues, les technosignes «pouce levé » et «pouce baissé » invitent l'auditoire à marquer son approbation ou au contraire à signifier sa désapprobation. Dans le blogue «Votre Tour du monde », c'est en cliquant à la fin d'un article sur un nombre d'étoiles (jusqu'à cinq) que les écrilecteurs peuvent évaluer le contenu du billet. Le nombre de votes ou de clics (pouce levé ou baissé) sont quantifiés et affichés. Ils sont une première forme de commentaires numériques et un 
premier moyen technodiscursif proposé par les interfaces aux écrilecteurs pour entrer en relation avec le blogueur ou le vlogueur et communiquer une appréciation sur le contenu proposé.

\section{2. Les commentaires}

Les commentaires numériques sont également des éléments caractéristiques de la structure relationnelle du technogenre du blogue ou du vlogue. Le blogueur ou le vlogueur a le choix d'ouvrir ou pas son article ou sa vidéo aux commentaires, toutefois, comme Crystal (2006) le souligne, quiconque restreindrait cette possibilité, sous-utiliserait sérieusement les fonctionnalités potentielles du blogue ou du vlogue. Paveau définit le commentaire numérique ainsi :

Structurellement lié au discours premier qu'il prédique, selon des modalités très diverses, y compris non langagières, il est avant tout un lieu de relationalité. Participant du mode de construction et de réception du sens du texte premier, il ressortit du processus d'écrilecture spécifique aux discours connectés. (2017:55)

Technodiscours second produit dans un espace dédié scripturalement et énonciativement contraint au sein d'un écosystème connecté, il est un technogenre à part entière et présente les traits discursifs suivants : l'énonciation pseudonyme, la relationalité, la conversationnalité et récursivité, l'augmentation énonciative et discursive, la publicité et visibilité (Paveau 2017).

Dans le blogue «Votre Tour du monde », à la fin de chaque article, l'écrilecteur est invité à écrire un commentaire. La page dans laquelle le blogueur se présente ( À propos $\left.{ }^{8}\right)$ a reçu à titre d'exemple, entre le 7 juillet 2015 et le 27 novembre 2018, 44 commentaires d'internautes auxquels Bruno, le blogueur, a fourni 17 réponses individuelles. Nous y retrouvons des commentaires-remerciements, des commentaires-encouragements, des commentaires-partages d'expérience personnelle, des commentaires-questions qui incitent le blogueur à répondre. L'article initial est donc ainsi augmenté, potentiellement à l'infini, la conversation n'étant jamais finie. Par ailleurs, l'augmentation se poursuit en dehors de l'article et même hors du blogue. Le blogueur par exemple, en guise de réponse à une question, renvoie vers un article traitant du sujet par lien intégré ou vers des posts d'autres «Voyageurs autour du Monde ${ }^{9}$ » dans le groupe Facebook afférent qu'il a créé.

\footnotetext{
${ }^{8}$ Voir en ligne : <https://www.votretourdumonde.com/a-propos-de-bruno/>.

${ }^{9}$ https://www.facebook.com/groups/tourdumondistes/
} 
Répondre

Salut Bruno ! Sympa de partager un témoignage de tes découvertes et pérégrinations. Pour avoir fait moi même, le tour du monde sur une longue période même en exerçant professionnellement, aujourd'hui à la retraite je ne me lasse pas de mes évasions. Bonne continuation .

Un Calédonien résidant en France

\section{Elodie}

Très cool! un rêve secret qui habite pas mal de personnes sur cette terre...mais comment fais-tu financièrement? Merci

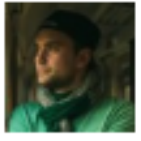

Votre Tour du Monde

Répondre

Je t'invite à aller lire cet article: https://www.votretourdumonde.com/digitalnomad/ $:$

Répondre

Salut,

Je compte partir en Inde bientôt ! tu pourrais peut être m'aider concernant les billets ouverts?

II me semble qu'il faut un billet de retour mais j'ai le visa 6 mois touriste et je ne sais absolument pas quand je rentre!

Si t'as des conseils concernant des compagnies aérienne, je te remercie d'avance!

Evy

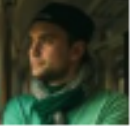

\section{Votre Tour du Monde}

Répondre

Je ne suis jamais allé en Inde mais tu peux poser ta question ici:

https//www.facebook.com/groups/tourdumondistes/ d'autres voyageurs pourront te répondre $\Theta$ 
Figure 8 : Commentaires sur le blogue

La plateforme YouTube, bien que définie comme site d'hébergement et de partage de vidéos, est davantage perçue par les utilisateurs comme un réseau social (Burgess \& Green 2009 ; Barton \& Lee 2013) en raison de la relation privilégiée entre ceux qui publient des vidéos et ceux qui les regardent et les commentent. La fonctionnalité «commentaires » offre donc la possibilité de nombreuses interactions avec d'autres internautes. Par ailleurs, selon la configuration de lecture des commentaires choisie, «Top des commentaires » ou «Les plus récents d'abord », l'écrilecteur verra son action orientée. En effet, «Top des commentaires » est une fonctionnalité proposée par YouTube qui permet d'afficher les commentaires selon un classement qui met en avant ceux du vlogueur, ceux suscitant des discussions auprès des spectateurs et ceux ayant enregistré des clics sur le technosigne «j'aime » au sein de la communauté ${ }^{10}$.

Dans le vlogue «Osons causer», la vidéo postée le 12 décembre 2018, «Si Macron vous a convaincu, regardez ça ${ }^{11}$ », totalise au 20 décembre $2018: 688435$ vues, 30K de pouce levés, 2,3K de pouces baissés et 3751 commentaires. Les commentaires suscitent eux-mêmes un nombre conséquent de polylogues : 151 réponses, 19 réponses, 55 réponses, 61 réponses, 30 réponses, 59 réponses, etc. Le fil discursif prolonge donc la vidéo initiale dans un continuum potentiellement infini, l'ensemble formant un tout technosémiodiscursif. Chaque commentaire peut lui aussi être apprécié par un pouce levé ou baissé et également commenté avec une adresse éventuelle (mais proposée à l'écriture par la plateforme) vers l'écrilecteur auquel il est dirigé. Le vlogue est ainsi un espace générateur d'échanges non seulement avec les vlogueurs mais aussi entre les internautes.

Dans l'exemple ci-après, nous observons un commentaire initial des vlogueurs, réponse à leur propre vidéo pour corriger une erreur de chiffres au sein de celle-ci. Ce commentaire suscite lui-même 151 réponses dont la capture d'écran ci-dessous est un extrait.

\footnotetext{
${ }^{10}$ Source : Aide YouTube, voir en ligne : <https://support.google.com/youtube/answer/6000976?hl=fr>.

${ }^{11}$ Voir en ligne : <https://youtu.be/FYJ3NUWoOvE>.
} 
Orons Causere ty a 1 semaine (modife)

ERRATUM : Ca ne change rien aux arguments de la vidéo (ISF + Flat tax coûte bien autant que les nouvelles mesures annoncées), mais nous avons fait une erreur d'inattention. Nous disons que la réforme fiscale fait gagner $18500 €$ par mois aux $1 \%$ les plus riches. En fait ils gagnent ces $18500 €$ sur une année ! Cela équivaut donc à $1550 €$ par mois ! Ce qui reste bien loin des 40 de prime d'activite : Mais nous présentons nos excuses pour cette erreur dinattention. On était visiblement un peu trop fatigués pour regarder notre vidéo "Macron offre une Porsche à 280.000 Français" qui a les bons chiffres.

16 725 RÉPONDRE

Masquer les réponses $\wedge$

Q

Matheo Kortez il y a 1 semaine

II faudrait peut-être refaire la vidéo, afin de ne pas être décrédibilisé ... ?

55 REPONDRE

(ง)

Julien il y a 1 semaine (modifié)

Osons Causer avec lerreur des $18500 €$ par mois au lieu de par an on peut plus partager ça. Ça vous décrédibilise pour qui sait ou apprend que ciest faux. Vous pouvez pas caser une note écrite en gros sur la vidéo a ce passage précis ??

Sinon a par ça cette vidéo c'est quasiment une copie du discours de Mathilde Panot, mais sans parler du référendum dinitiative populaire (RIC).

44 REPONDRE

Gérard Galaxy il y a 1 semaine

Merci pour la rectification. Mieux vaut être précis, mais peu importe la somme, ça fait longtemps que je compte plus qu'en seaux de vomi.

16 22 REPONDRE

C.

Finitude WoW ily a 1 semaine

C'est quand même toujours mieux que 40 euros

17 GI RÉPONDRE

Il s'apprête à supprimer les revenus maladies sur la base du 'faites du télétravail'.

16 5 \%I REPONDRE

A Alister one il y a 1 semaine (modifie)

Bonjour, jaimerais vous partager une réflexion qui fait sont chemin en ce moment, plus ont voir macron plus ont s'imagine quill est incompétent....... je ne pense pas qu'a ce niveaux là c'est de l'incompétence ou du mépris.

Il devient important d'imaginer quili à quelque chose de prévu pour nous et que le chaos quil met est voulu, comme la vente des aéroports de Paris, ou la suppression de la taxe sur les pub pour les chaines de TV.

https://www youtube com/watch?v= J18zukKyDzs

dans cette vidéo cette personne expose un certain nombre de chose dont notamment des moyens d'action contre ceux qui nous gouverne.

Jaimerais aussi vous demander dêtre prudent quand vous manifestez, quand ont cherche à imaginer jusqu'où ces gens sont prêt à allez, ont peux difficilement ne pas imaginer que répandre notre sang est ce quill cherche.

bon courage à vous j'espère que la situation riest pas aussi dingue qu'elle en à l'air.

Moins

14 $6 \quad$ qI REPONDRE

(9)

(Alister one Il faut que tu comprennes quil gère la France comme une banque et le but d'une banque c'est de gagner des profits

14. 8 REPONDRE

Pixar Burton il y a 1 semaine

Osons Causer Un tit montage pour corriger ça surtout dans le contexte actuel, j'ai deja diffusé cette video partout ;-)

14 5 TI REPONDRE

Zack Angel il y a 1 semaine

fapprouve pour une nouvelle vidéo pour corriger lérreur svp

16 11 \% RÉPONDRE

Figure 9 : Commentaires sur le vlogue

Nous y observons un technocommentaire-réponse en bleu à un autre internaute @ Alister one qui le distingue des autres commentaires-réponses a priori destinés à « Osons Causer » que l'indentation à droite indique comme tel. Dans ce bref extrait, nous distinguons également plusieurs types de commentaires: le commentaire-remerciement «Merci pour la rectification... », le commentaire-lien ${ }^{12}$ (lien vers une autre vidéo YouTube), le commentaire discursif « Il s'apprête à supprimer les revenus maladies... » qui vient prolonger le contenu de la vidéo. Le sujet étant politique et se prêtant à la polémique, plusieurs commentaires sont susceptibles de relever du commentaire-troll comme par exemple «Wesh wesh ${ }^{13}$ les communistes » ou «Ton sourire de bourgeois qui prétend aider les pauvres me donne une envie ardente de t'en coller une». Enfin, nous notons le commentaire métadiscursif «Il

\footnotetext{
${ }^{12}$ Modifié depuis, le lien vers la vidéo a notamment disparu.

${ }^{13}$ En écho au rituel d'ouverture de Ludo qui commence généralement ses vidéos par « Wesh wesh les amis ».
} 
faudrait peut-être refaire la vidéo, afin de ne pas être décrédibilisé..? » qui encourage les vlogueurs à refaire cette vidéo, celle-ci ayant été notamment partagée par les écrilecteurs «...j j'ai déjà diffusé cette vidéo partout ;-) ». C'est non seulement la crédibilité des vlogueurs mais également des écrilecteurs qui se trouvent engagée, ceux-ci l'ayant eux-mêmes partagée dans leur réseau sous leur pseudonyme en technodiscours rapporté. Dans cet exemple particulier, ce type de commentaire n'est pas isolé («j'approuve pour une nouvelle vidéo pour corriger l'erreur svp », «Je plussoie, il faut reposter la vidéo corrigée, car sinon on va se faire taxer de gauchistes manipulateurs diffuseurs de fake news :) ») et met bien en évidence la notion de communauté sociotechnodiscursive que le technogenre génère, comme nous allons l'évoquer.

\section{3. La communauté sociotechnodiscursive}

Étudiées depuis la fin des années 1990, les communautés alors appelées virtuelles «sont des regroupements socioculturels qui émergent du réseau lorsqu'un nombre suffisant d'individus participent à ces discussions publiques pendant assez de temps en y mettant suffisamment de cœur pour que des réseaux de relations humaines se tissent au sein du cyberespace » (Marcoccia $2016: 139)$.

Toutefois, il ne suffit pas que des messages soient échangés sur Internet par des individus pour que ceux-ci forment une communauté. Plusieurs indicateurs ont été retenus pour affirmer qu'un groupe conversationnel en ligne se constitue effectivement en communauté comme un sentiment d'appartenance, des activités de parole ritualisées, une identité communautaire, un système de coopération entre les membres, le partage de valeurs et de buts communs, la durée des échanges, une forme d'organisation sociale (Marcoccia 2016). Crystal (2006) insiste également sur les particularités linguistiques des communautés en ligne et parle d'un sociolecte que les nouveaux arrivants devront apprendre s'ils veulent se joindre à la communauté. Les caractéristiques du technogenre contribuent même à la formation de la communauté. Crystal (2006) parlait déjà des blogging communities et soulignait l'importance des hyperliens, précisant que plus un site est lié à d'autres sites, plus il est présent et donc plus il a d'influence et d'autorité.

Dans le cas du blogue ou du vlogue, c'est tout d'abord dans un espace commun partagé, la blogosphère ou vlogosphère, que les blogueurs ou les YouTubeurs se retrouvent. C'est ensuite autour de la thématique comme le «blogue de voyage» ou «le vlogue politique » que la communauté peut se créer, les internautes se dirigeant spontanément vers tel ou tel blogue ou vlogue en fonction de leurs centres d'intérêt ou encore tel ou tel blogue ou vlogue leur étant directement suggéré par les interfaces sur lesquelles ils naviguent en fonction de précédentes recherches. Toutefois, par rapport aux autres communautés en ligne, c'est aussi principalement autour de la personne d'un blogueur ou d'un vlogueur que cette communauté peut se créer. Les chiffres, même s'ils ne nous indiquent pas la qualité du lien qui s'est tissé, nous renseignent sur le nombre d' «amis » ou d'abonnés et l'on constate par exemple que le blogueur Bruno de «Votre Tour du Monde», présente des chiffres importants pour un anonyme en termes d'abonnés à son compte Twitter (14k), à sa page Facebook (194k), à son groupe Facebook (123 378 membres), à sa chaîne YouTube (57 236) ou à son compte 
Instagram $(165 \mathrm{k})^{14}$. Un internaute (qui n'est pas unique) précise d'ailleurs à Bruno en commentaire : «Je te suis pratiquement sur tout (sic) les réseaux sociaux ».

Fédérer une communauté ne va cependant pas de soi. En ce qui concerne les vlogues, nous avions observé dans une étude exploratoire que les vlogueurs optent pour une mise en scène travaillée destinée à attirer les abonnés, mise en scène où le titre et le cadrage jouent un rôle essentiel, que l'ouverture et la clôture jouent un rôle socio-affectif important et servent à tisser des liens avec les destinataires, que ce lien affectif tend à encourager l'abonnement à la chaîne. Et c'est le tissage de ces liens d'abord entre le vlogueur et son public qui peut aboutir finalement à la création d'une communauté de fans autour de ce même vlogueur et de la thématique qu'il développe (Combe Celik 2014). Barton et Lee (2013) affirment également que les vlogueurs considérant leurs spectateurs et leurs abonnés comme leur capital social ont tendance à adopter des identités ludiques afin d'attirer davantage de spectateurs et d'abonnés.

Dans nos deux exemples, nous observons la volonté du blogueur et des vlogueurs d'inclure leurs destinataires dans leur discours, comme le suggèrent le titre «Votre Tour du Monde » et son extension «Le blog voyage qui vous inspire! » ou encore la marque de la première personne du pluriel dans le pseudo des vlogueurs «Osons causer» et l'adresse ritualisée «Les amis » que Ludo d' « Osons causer » formule à destination de l'auditoire. Ils cherchent à former une communauté autour d'une thématique qui les passionne et n'hésitent pas à susciter la parole de leurs destinataires comme lorsque Bruno interviewe d'autres internautes voyageurs et poste sur son blogue leurs expériences ou lorsque le collectif «Osons causer » enregistre à trois cette fois-ci une vidéo $\mathrm{FAQ}^{15}$ dans laquelle ils répondent aux questions posées en direct par les internautes sur les réseaux sociaux. Cette volonté de communauté, dépassant même l'univers numérique, est aussi clairement énoncée par cette même équipe sur leur site : «Enfin, pour casser l'asymétrie du dispositif, nous essayons de le rendre le plus horizontal possible, de donner beaucoup et de tisser une relation avec notre public. Nous répondons aux commentaires de la manière la plus constructive possible. Et organisons (bientôt) régulièrement des rencontres-débats joyeuses avec notre public et des émissions de radio. »

C'est pourquoi nous préférons adopter le terme de communauté "sociotechnodiscursive », c'est-à-dire une communauté qui se forme au sein d'un espace numérique privilégié comme la blogosphère ou la plateforme YouTube, autour d'un intérêt partagé pour une thématique et dont les liens, qu'ils soient plus ou moins faibles ou forts entre les internautes, sont principalement technodiscursifs.

\section{Conclusion : vers une con-fusion des genres ?}

Dans cet article, nous avons cherché à montrer les caractéristiques de deux technogenres prescrits, emblématiques du web social, le blogue et le vlogue, à partir de deux exemples, un blogue de voyage sur Wordpress et un vlogue d'éducation politique et citoyenne sur

\footnotetext{
${ }^{14}$ Chiffres recueillis à la date du 20/12/2018.

${ }^{15}$ « OSONS CAUSER FAQ 3 ANS + CHAÎNES QU'ON AIME », voir en ligne : $<$ https://youtu.be/nuk963WVAlo>.
} 
YouTube. Après avoir observé comment l'énonciation numérique se réalisait au sein du CMS Wordpress et de la plateforme YouTube, nous avons envisagé la structure relationnelle du blogue et du vlogue d'une part entre les technodiscours et d'autre part entre écrilecteurs, les deux étant éminemment liés.

En conclusion, il apparaît que la structure relationnelle du blogue et du vlogue part du microtechnodiscours de l'énonciateur numérique qu'est l'article du blogue ou la vidéo du vlogue pour s'étendre à l'ensemble du blogue et de la chaîne YouTube, jusqu'à l'univers macrotechnodiscursif du blogueur et du vlogueur au sein de ses réseaux sociaux pour enfin appartenir à une communauté sociotechnodiscursive plus vaste encore qui se forme autour du blogue et du vlogue et de ses créateurs, comme l'illustre la figure 10 ci-dessous.

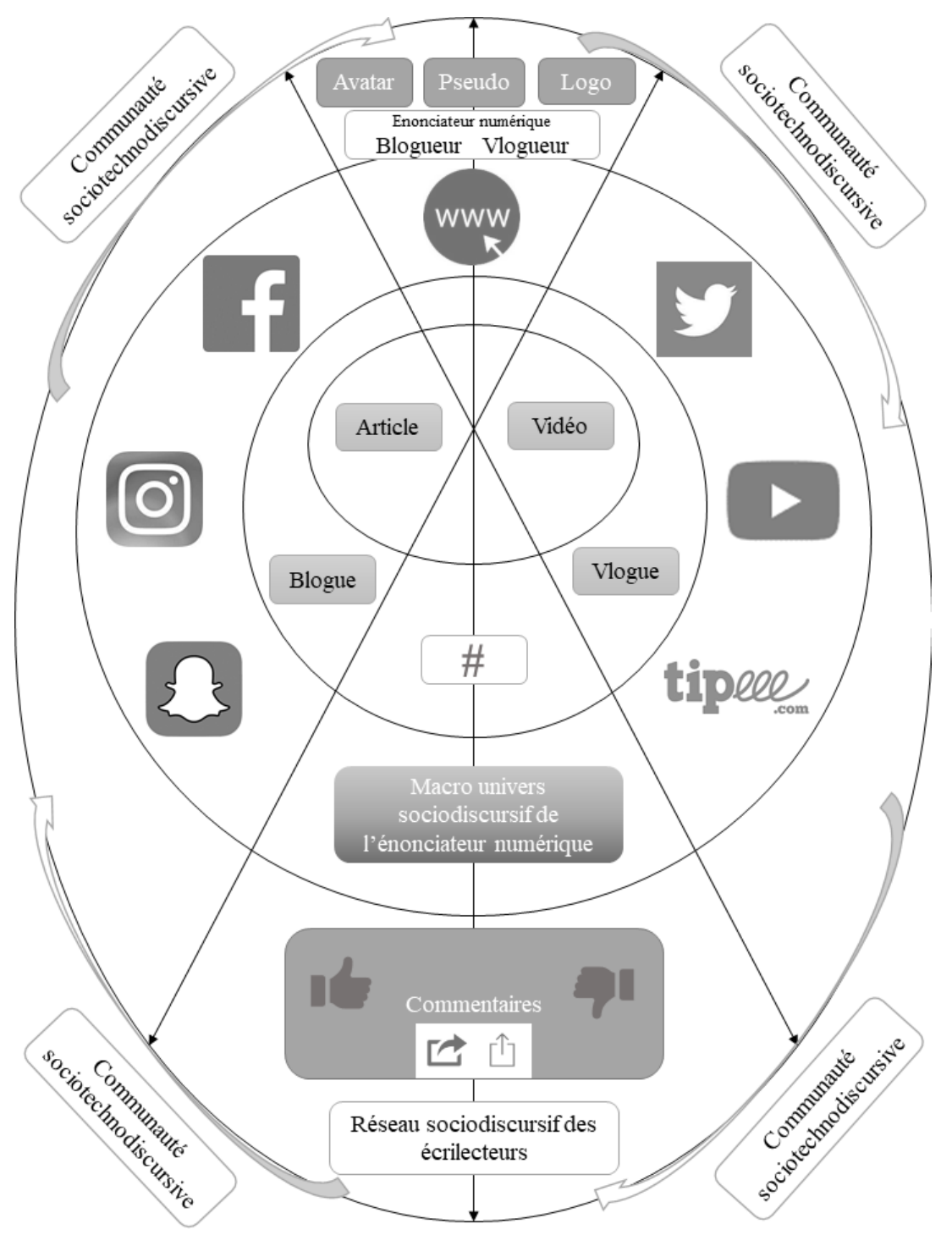

Figure 10 : Structure relationnelle du blogue et du vlogue 
Et, même s'ils se développent au sein de plateformes différentes, le blogue et le vlogue présentent les caractéristiques communes du «genre de la relation » que nous nous proposons de définir de la manière suivante.

Dans un genre de la relation, le locuteur principal se met en scène par des pratiques extimes qui vont de la narration de soi à la première personne à l'autoportrait ou l'autoscopie dans son environnement intime. Il y a un engagement fort de ce locuteur auteur pour fédérer ses interlocuteurs par des termes d'adresse mais aussi en leur donnant l'opportunité d'interagir avec lui par des boutons d'appréciation (comme des pouces levés ou baissés ou des étoiles) et par des espaces d'échanges plus longs (comme des commentaires). Dans un genre de la relation, les échanges peuvent dépasser le simple dialogue entre le locuteur auteur et les membres intéressés pour se transformer en un vaste polylogue entre membres de la même communauté sociotechnodiscursive. Un genre de la relation se caractérise également par une porosité des contenus qui circulent entre les différentes plateformes qui viennent parfois ellesmêmes s'entremêler.

Pour finir, nous ne mettrons pas de point final à l'étude descriptive des genres numériques, genres qui nous semblent en perpétuelle évolution. Les plateformes tendent non seulement à devenir de plus en plus plurisémiotiques, interactives et multimédiatiques, mais également à s'uniformiser. Le blogue que l'on définissait précédemment par des billets organisés antéchronologiquement se confond désormais avec la notion de site, site où l'image est de plus en plus prédominante (pictorial turn), où les vidéos YouTube sont directement intégrées. Les espaces de production discursive du CMS ou de la plateforme interagissent toujours davantage avec l'écrilecteur devenant des espaces de plus en plus complexes de création multimédiatique, de gestion à la fois technique, sociale et discursive (voir figures 3 et 5). Plus que jamais, l'internaute du $\mathrm{XIX}^{\mathrm{e}}$ siècle est conduit à développer des compétences toujours plus grandes en littératie numérique pour évoluer (au sens propre comme au sens figuré) au sein des univers connectés.

\section{Références bibliographiques}

Bakhtine M. (1984), Esthétique de la création verbale, Paris, Gallimard.

Barton D. \& Lee C. (2013), Language Online: Investigating Digital Texts and Practices, Londres/New-York, Routledge.

Bibié-Emerit L. (2018), «La préconstruction technolangagière dans les souhaits d'anniversaire sur Facebook», dans Mezzadri A. et al. (dir.), Le préconstruit approche pluridisciplinaire, Paris, Classiques Garnier, p. 211-229.

Burgess J. \& Green J. (2009), YouTube: Online video and participatory culture, Cambridge Polity Press.

Candel E. (2010), «Penser la forme des blogs, entre générique et génétique », Itinéraires. Littérature, textes, cultures 2, p. 23-31.

Casilli A. (2013), "Contre l'hypothèse de la "fin de la vie privée". La négociation de la privacy dans les médias sociaux », Revue française des sciences de l'information et de la 
communication 3. En ligne $:\langle$ http://journals.openedition.org/rfsic/630>, consulté le 14/02/2019.

Combe Celik C. (2014), «Vlogues sur YouTube: un nouveau genre d'interactions multimodales », dans Colon de Carjaval I. \& Ollagnier-Beldame M. (dir.), Actes du colloque Interactions Multimodales Par Ecrans 2014, Lyon, du 2 au 4 juillet 2014. En ligne: <https://impec.sciencesconf.org/conference/impec/pages/Impec2014_Combe_Celik.pdf>, consulté le 14/02/2019.

Combe C. (2016), «Questions méthodologiques autour de l'étude de deux plateformes interactives multimodales : de la communauté de contenu à l'application intimiste », Línguas e Instrumentos Linguítiscos $37 . \quad$ En ligne : <http://www.revistalinguas.com/edicao37/artigo9.pdf>, consulté le 14/02/2019.

Combe C. (2017), «Télécollaboration informelle 2.0 : le vlogue d'un américain en français sur YouTube », Alsic 20(2). En ligne : <http://journals.openedition.org/alsic/3094>, mis en ligne le 26/06/2017, consulté le 14/02/2019.

Combe C. (à paraître en 2019), «Quand le vlogue éduque à la politique : le cas français d'Osons causer », dans George É. (dir.), Numérisation généralisée de la société et enjeux sociopolitiques de la culture et de la communication, Londres, Éditions ISTE.

Côté J. (2012), «Les enjeux éthiques de l'utilisation d'Internet en recherche: principale questions et pistes de solutions », Éthique publique 14(2). En ligne : <http://ethiquepublique.revues.org/997>, consulté le 14/02/2019.

Crystal D. (2006), Language and the Internet, Cambridge, CUP.

Deseilligny O. (2008), « Du journal intime au blog: quelles métamorphoses du texte ? », Communication \& Langages 155, p. 45-62. En ligne : <http://www.persee.fr/doc/colan_03361500_2008_num_155_1_5374>, consulté le 14/02/2019.

Develotte C. (2006), « Décrire l'espace d'exposition discursive dans un campus numérique », Le Français dans le monde, Recherches et applications, p. 88-100.

Develotte C. (2012), «L'analyse des corpus multimodaux en ligne : état des lieux et perspectives », dans «SHS Web of Conferences 1 », EDP Sciences, p. 509-525. En ligne : $<$ https://www.linguistiquefrancaise.org/articles/shsconf/pdf/2012/01/shsconf_cmlf12_000213. pdf>, consulté le 14/02/2019.

Doueihi M. (2011), Pour un humanisme numérique, Paris, Seuil.

Flichy P. (2014), Le Sacre de l'amateur. Sociologie des passions ordinaires à l'ère numérique, Paris, Seuil.

Georges F. (2009), «Représentation de soi et identité numérique. Une approche sémiotique et quantitative de l'emprise culturelle du web $2.0 »$ Réseaux 154, p. 165-193. 
Granjon F. (2012), Reconnaissance et usages d'internet. Une sociologie critique des pratiques de l'informatique connectée, Paris, Presses des Mines.

Herring S. C. (2015), «New frontiers in interactive multimodal communication », dans Georgakopoulou A. \& Spilioti T. (dir.), The Routledge Handbook of Language and Digital Communication, London, Routledge, p. 398-402.

Herring S. C., Scheidt L. A., Wright E. \& Bonus S. (2005), «Weblogs as a bridging genre », Information Technology \& People 18(2), p. 142-171.

Jeanne-Perrier V. (2006), «Des outils d'écriture aux pouvoirs exorbitants ? », Réseaux 137, p. 97-131.

Jeanneret Y. \& Souchier E. (1999), «Pour une poétique de "l'écrit d'écran" », Xoana, Images et sciences sociales 6, p. 97-107.

Jeanneret Y. \& Souchier E. (2005), «L'énonciation éditoriale dans les écrits d'écran », Communication \& langages 145(1), p. 3-15.

Labbé H. \& Marcoccia M. (2005), «Communication numérique et continuité des genres : l'exemple du courrier électronique », Texto 10(3). En ligne: <http://www.revuetexto.net/Inedits/Labbe-Marcoccia.html>, consulté le 14/02/2019.

Leadbeater C. \& Miller P. (2004), The pro-am revolution. How enthusiasts are changing our economy and society, London, Demos.

Maingueneau D. (2002), «Typologie des discours », dans Charaudeau P. \& Maingueneau D. (dir.), Dictionnaire d'analyse du discours, Paris, Seuil.

Maingueneau D. (2013), «Genres de discours et web : existe-t-il des genres web ? », dans Barats C. (dir.), Manuel d'analyse du web en Sciences Humaines et Sociales, Paris, Armand Colin, p. 74-93.

Maingueneau D. (2016), Les termes clés de l'analyse du discours, Paris, Seuil.

Marcoccia M. (2016), Analyser la communication numérique écrite, Paris, Armand Colin.

Mourlhon-Dallies F., Reboul-Touré S. \& Rakotonoelina F. (dir.) (2004), «Les discours de l'internet : nouveaux corpus, nouveaux modèles ? », Carnets du Cediscor 8.

Paveau M.-A. (2013), «Genre de discours et technologie discursive », Pratiques 157-158, p. 7-30.

Paveau M.-A. (2015), «Ce qui s'écrit dans les univers numériques. Matières technolangagières et formes technodiscursives », Itinéraires. Littérature, textes, cultures. En ligne : <https://journals-openedition-org.lama.univ-amu.fr/itineraires/2313\#tocto2n4>, consulté le 14/02/2019. 
Paveau M.-A. (2017), L'analyse du discours numérique. Dictionnaire des formes et des pratiques, Paris, Hermann.

Thély N. (2012), «La web-intimité », Raison-Publique.fr arts • politique • société [site]. En ligne : <http://www.raison-publique.fr/article542.html>, consulté le 14/02/2019. 